\title{
Therapie der Mukoviszidose in den ersten beiden Lebensjahren
}

\section{Der richtigen Behandlung nach früher Diagnose kommt große Bedeutung zu.}

\begin{abstract}
Seit der Einführung des Neugeborenen-Screenings auf Mukoviszidose im Jahr 2016 werden betroffene Kinder deutlich früher diagnostiziert, und der richtigen Behandlung nach dieser frühen Diagnose kommt eine besondere Bedeutung zu. Nach fast sechsjähriger Entwicklungsphase hat nun eine Expertengruppe um PD Dr. Lutz Nährlich, Universitätsklinikum Gießen/Marburg, und Dr. Jutta Hammermann, Universitätsklinikum Dresden eine S3-Leitlinie zur Mukoviszidose-Behandlung in den ersten beiden Lebensjahren veröffentlicht. Dies ist die erste deutsche Leitlinie für diese Patientengruppe.
\end{abstract}

\section{Leitlinie umfasst verschiedene Themengebiete}

Mit der Leitlinie liegen erstmals umfassende Empfehlungen für die Frühtherapie der Mukoviszidose (Cystische Fibrose - CF) vor, die sowohl die Lungenerkrankung als auch die Erkran-

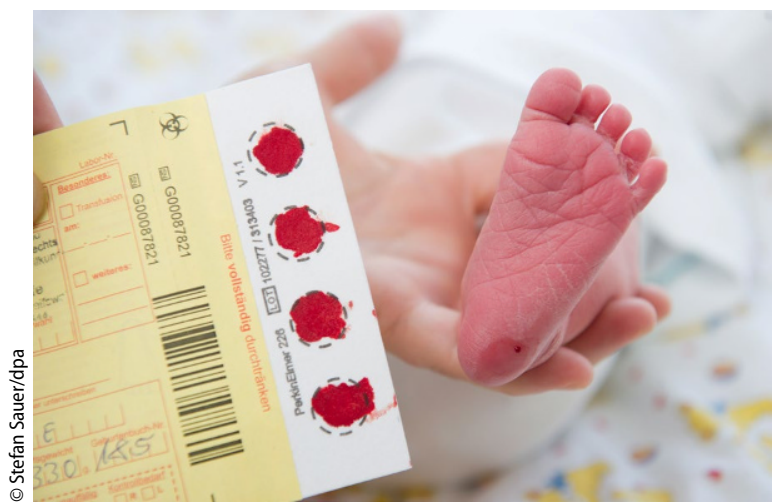

A Seit 2016 werden auch in Deutschland Neugeborene routinemäßig auf Mukoviszidose untersucht sichtigen. Die richtige Ernährung und die Physiotherapie spielen ebenfalls eine große Rolle. Das erste Kapitel beschäftigt sich mit allgemeinen Empfehlungen wie z. B. zur Diagnoseeröffnung, zur Zusammenarbeit zwischen spezialisierten CF-Einrichtungen und dem Kinderarzt oder zu Schulungen und Beratungsangeboten.

Die Leitlinie wurde von der Arbeitsgemeinschaft der Wissenschaftlichen Medizinischen Fachgesellschaften (AWMF) geprüft und in ihre Leitliniendatenbank aufgenommen. Der Leitlinientext sowie alle zugehörigen Dokumente einschließlich des detaillierten Reports zur methodischen Vorgehensweise sind frei verfügbar. Die Erarbeitung einer laienverständlichen Patientenversion ist geplant.

\section{Wichtigste Empfehlungen in der Übersicht}

Zusätzlich zum eigentlichen Leitlinientext stellen die Autoren auch eine Übersichtstabelle zur Verfügung, die sich an internationale Standards anlehnt und in der die wichtigsten Empfehlungen im zeitlichen Verlauf von der Diagnose bis zum zweiten Lebensjahr dargestellt sind - eine wertvolle Ergänzung im klinischen Alltag. Trotzdem lohnt die Lektüre der vollständigen Leitlinie: neben den Hintergründen zu den Empfehlungen sind dort auch weitere Aspekte, wie z.B. nicht empfohlene Behandlungsmethoden aufgeführt.

\section{Nach höchsten Qualitäts- standards entwickelt}

35 Experten aus verschiedenen Berufsgruppen und Patientenver- treter sowie 18 Fachgesellschaften und Berufsverbände haben unter Federführung von GPP (Gesellschaft für Pädiatrische Pneumologie) und DGKJ (Deutsche Gesellschaft für Kinder- und Jugendmedizin) an der Entstehung der neuen Leitlinie mitgewirkt. Erstellt wurde die Leitlinie auf S3-Niveau, der höchsten Qualitätsstufe von Leitlinien. Die Erarbeitung erfolgte unabhängig von einer Einflussnahme durch die Industrie, etwaige Interessenskonflikte der Beteiligten wurden angegeben und ausgewertet. Das Mukoviszidose Institut hat die Entwicklung mit personellen Ressourcen bei Organisation und Leitlinienmethodik unterstützt.

\section{Weitere Informationen:}

Leitlinie zur Mukoviszidose-Frühtherapie in der Leitliniendatenbank der AWMF einsehen: https://www.awmf.org/leitlinien/detail/l//026024.html

Hinweis des Verlags. Der Verlag bleibt in Hinblick auf geografische Zuordnungen und Gebietsbezeichnungen in veröffentlichten Karten und Institutsadressen neutral.

Paediatr. Paedolog. 2020 · 55:222 https://doi.org/10.1007/s00608020-00823-z

(c) Springer-Verlag GmbH Austria, ein Teil von Springer Nature 2020 